\title{
Structural Changes in the Posterior Interosseous Nerve from Patients with Wrist Osteoarthritis and Asymptomatic Controls
}

\author{
${ }^{1}$ Department of Clinical Science and Education, Karolinska Institutet, \\ Södersjukhuset, Stockholm, Sweden \\ 2 Department of Laboratory Medicine, Division of Pathology, \\ Karolinska Institutet, Karolinska University Hospital, Stockholm, \\ Sweden
}

Elin Swärd, MD ${ }^{1}$ Inger Nennesmo, MD, $\mathrm{PhD}^{2} \quad$ Maria Wilcke, MD, PhD ${ }^{10}$

J Wrist Surg 2020;9:481-486.

\begin{abstract}
Keywords

- myelinated fiber

- nerve morphology

- peripheral nerve

- posterior interosseous nerve

- wrist osteoarthritis

Background Posttraumatic morphological changes have been described in the posterior interosseous nerve (PIN) after mild wrist trauma, and it has been suggested that posttraumatic nerve changes may contribute to wrist pain. PIN excision has shown to relieve pain in some patients with wrist osteoarthritis. However, is not known if PINs from osteoarthritic wrist have pathological features.

Objective The aim of this study was to investigate whether PINs from osteoarthritic wrists show morphological changes that are not present in healthy wrists.

Materials and Methods PINs resected from 15 osteoarthritic wrists were analyzed with light microscopy regarding morphological changes and compared with five asymptomatic controls without osteoarthritis.

Results No significant differences in fascicular area, myelinated fiber density or myelinated fiber diameter were found. However, most patients and controls exhibited some degree of pathology, and a few samples from both groups exhibited severe pathological changes.

Conclusions Our findings of morphological changes in both patients with osteoarthritis and asymptomatic controls suggest that pathological changes of unknown significance might exist in the general population in the PIN at wrist level. We believe that the observed structural nerve changes in the PIN are unlikely to contribute to the symptoms of pain. Further studies of the normal histological appearance of the terminal PIN are needed.

Level of Evidence This is Level II study.
\end{abstract}

Address for correspondence Maria Wilcke, MD, PhD, Handkirurgiska kliniken Södersjukhuset, Sjukhusbacken 10, Stockholm, 11883, Sweden (e-mail: maria.wilcke@sll.se).
The terminal branch of the posterior interosseous nerve (PIN) innervates the dorsal wrist capsule. ${ }^{1-5}$ PIN resection has shown to relieve pain in patients with posttraumatic wrist osteoarthritis ${ }^{6}$ and can be used for partial wrist denervation as a primary method for analgesia ${ }^{6,7}$ or in adjunction with surgical salvage procedures. ${ }^{8,9}$ In operations, where there is a risk of damaging the nerve through the surgical exposure, the PIN is often resected to prevent neuroma formation at wrist joint level. ${ }^{10}$

Morphological changes in the PIN at wrist level has been reported in patients with pain after repetitive dorsiflexion maneuvers of the wrist ${ }^{11}$ and after wrist sprains, ${ }^{7}$ indicating received

March 20, 2020

accepted

May 13, 2020

published online

July 29, 2020
Copyright $\odot 2020$ by Thieme Medical Publishers, Inc., 333 Seventh Avenue, New York, NY 10001, USA. Tel: +1(212) 760-0888.
DOI https://doi.org/ 10.1055/s-0040-1713655. ISSN 2163-3916. 
that structural changes may occur in the PIN after stress. No radiological pathology or signs of ligament disruption that could explain the symptoms of pain were found in these studies. It is not known if resected PINs from osteoarthritic wrists have pathological features.

The aim of this study was to investigate whether PINs from osteoarthritic wrists show morphological changes that are not presents in asymptomatic wrists.

\section{Materials and Methods}

\section{Patients}

Fifteen patients with painful wrist osteoarthritis, where a salvage procedure (denervation or partial wrist fusion) with PIN resection was planned, were included. As controls, five PINs resected from formerly asymptomatic wrists with acute conditions such as distal radius fractures or perilunar wrist dislocations were included. Exclusion criteria were a history of neuropathy, diabetes mellitus, rheumatoid or other inflammatory arthritis, alcoholism, drug abuse, amyloidosis, or other conditions that may affect the nerve. Controls with acute trauma 1 week or more ago, previous wrist pain, previous wrist trauma or reported previous wrist surgery were excluded.

All patients were operated through a standard dorsal approach. Loupe magnification $(\times 3.5)$ was used. The 1.5 to $2 \mathrm{~cm}$ long PIN biopsies were taken at the proximal border of the extensor retinaculum or in the proximal part of the fourth extensor compartment.

\section{Biopsies}

The nerve biopsies were fixed in $10 \%$ buffered formaldehyde. After 1 to 2 days, a piece of the nerve was taken from the central part of the specimen for further fixation in $2.5 \%$ glutaraldehyde for 2 days before Epon plastic embedding. From this plastic embedded material transverse $0.5-\mu \mathrm{m}$ thick sections were stained with $1 \%$ toluidine blue and processed for light microscopy (-Fig. 1). The remaining material was embedded in paraffin. Longitudinal and transverse sections, stained with hematoxylin and esosin, were thereafter analyzed.

The nerve biopsies were analyzed regarding presence of perineural thickening, endoneural fibrosis, inflammation, mucoid degeneration, abnormal appearance and distribution of myelinated fibers, and abnormal density of myelinated fibers, the latter as a sign of fiber loss.

The Epon plastic embedded sections were photographed at different magnifications using an Olympus BX53 light microscope equipped with a CAM-E3CMOS6.3 camera. Fascicle area was photographed and measured at $\times 20$ magnification and myelinated axon diameter and fiber density were measured at $\times 100$ magnification. The image analysis program Image J version 2.0 (http://imagej.nih.gov/ij) was used for all measurements. The sectional area from all but one nerve (one specimen
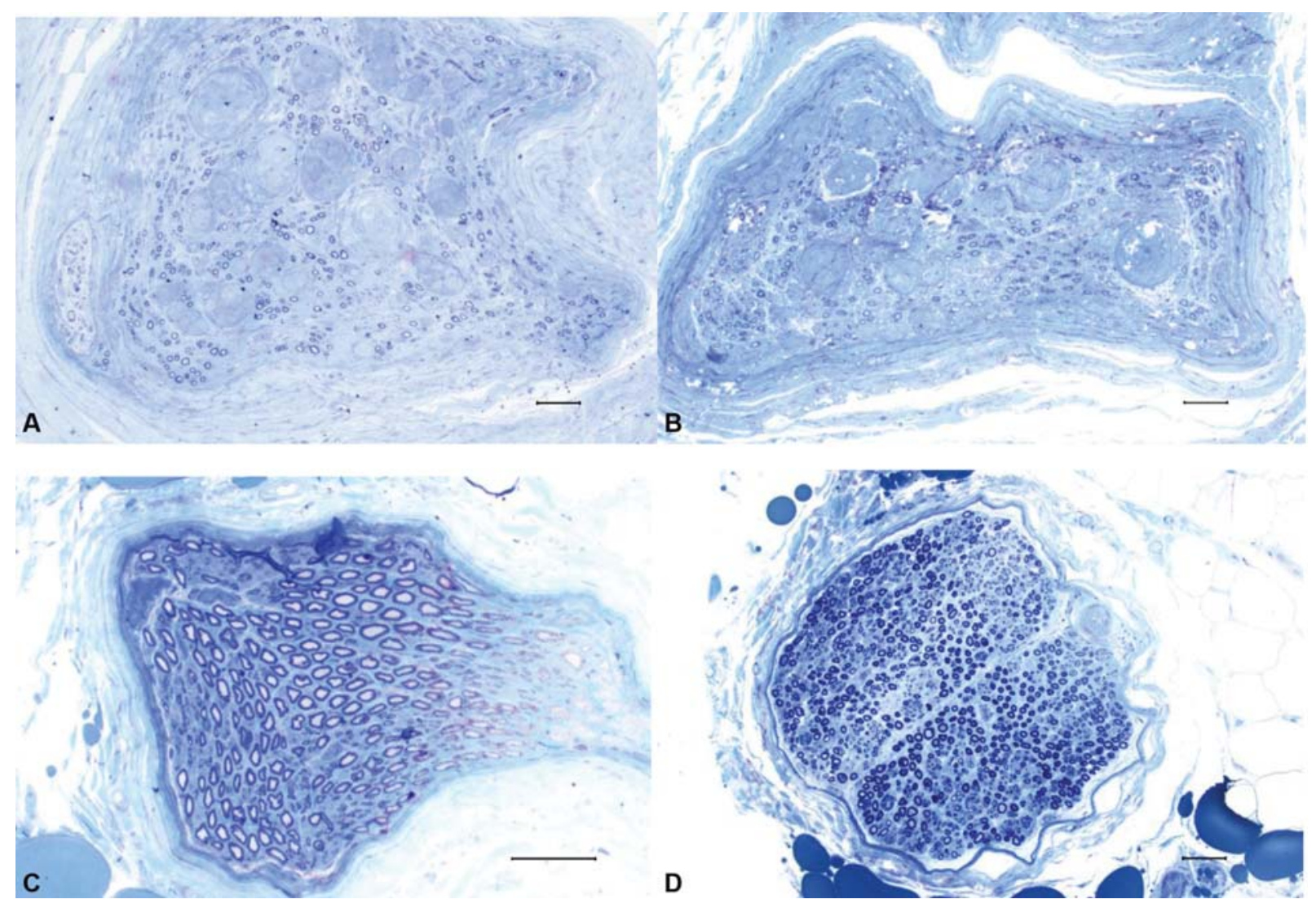

Fig. 1 PIN, semithin $(0.5 \mu \mathrm{m})$ transverse Epon-embedded sections, stained with toluidine blue, bar $50 \mu \mathrm{m}$. (A) Patient, 69 years old, severe structural changes (thickened perineurium, loss of myelinated fibers, and intraneural amorphous bodies of unknown origin). (B) Control, 55 years old, severe structural changes (thickened perineurium, loss of myelinated fibers, and intraneural bodies of unknown origin). (C) Control, 18 years old, minor structural changes. (D) Patient, 31 years old, minor structural changes. 
was damaged during preparation) was measured, and median values were derived. Only areas, where the specimen had been sectioned transversely and where all myelinated fibers had a circular or ovoid form, were considered to determine the diameter and density of myelinated nerve fibers.

Myelinated fibers were counted in a mean area of $0.4 \mathrm{~mm}^{2}$ per nerve (number of fibers counted 97-514). The fiber density for each sample was calculated dividing the number of fibers by the area where fibers had been counted (number $/ \mathrm{mm}^{2}$ ). The fiber diameters were determined by measuring the perimeter of each fiber (73-116 fibers were measured per nerve specimen), and the diameters were then calculated by Image $\mathrm{J}$.

The specimens were coded and an experienced neuropathologist (I.N.) performed the qualitative analysis. The quantitative analysis was performed by E.M.S., and a repeated blinded quantitative analysis was performed by M.K.W. The interrater reliability was excellent (interclass correlation coefficient 0,996 (CI 0.970-0.999).

\section{Statistical Analysis}

All data are presented as median and interquartile range (IQR) if not stated otherwise. Normality was tested with Shapiro-Wilks test. The Kruskal-Wallis test and MannWhitney $U$-test was used for comparison between groups. Results were considered significant at $p \leq 0.05$.

\section{Ethical Considerations}

Biopsies were stored according to the Swedish Act Biobanks in Medical Care (SFS 2002:297). Informed consent was obtained from each patient. The study was approved by the local Ethics Committee of Stockholm (DN 2017/2244-31).

\section{Results}

\section{General Results}

The patients with wrist osteoarthritis were significantly older than the control subjects $(p=0.008$, Mann-Whitney $U$-test). Concomitant medical conditions, such as cardiovascular or pulmonary diseases, were more common among the patients with wrist osteoarthritis. The characteristics of patients and controls are presented in - Table 1. There was no significant difference between patients and controls regarding fascicular sectional area, myelinated fiber density and myelinated fiber diameter. Morphological data are presented in - Table 2. Fiber diameter peaked at 4 to $5 \mu \mathrm{m}$ in both patients and controls (-Fig. 2). Specimens from two patients and two controls exhibited round amorphous intraneural bodies of unknown origin (-Fig. 1A, B).

\section{Controls}

All control specimens exhibited varying degrees of perineural thickening and mucoid degeneration. In three specimens these changes were mild (-Fig. 1C). In two samples more severe changes, such as loss of myelinated fibers (mean fiber density 2,139 and $2,595 / \mathrm{mm}^{2}$ respectively), endoneural fibrosis and abnormally thin myelin sheets in relation to
Table 1 Characteristics of patients with wrist arthritis and healthy controls

\begin{tabular}{|l|l|l|l|}
\hline \multicolumn{2}{|l|}{ Characteristics } & $\begin{array}{l}\text { Patients } \\
(\boldsymbol{n}=\mathbf{1 5})\end{array}$ & $\begin{array}{l}\text { Controls } \\
(\boldsymbol{n}=\mathbf{5})\end{array}$ \\
\hline $\begin{array}{l}\text { Age (y) } \\
\text { Median (range) }\end{array}$ & $61(25-79)$ & $22(18-55)$ \\
\hline \multirow{4}{*}{ Gender } & Male & 12 & 3 \\
\cline { 2 - 4 } & Female & 3 & 2 \\
\hline Profession & Manual work & 10 & 3 \\
\cline { 2 - 4 } & Sedentary work & 1 & 1 \\
\cline { 2 - 4 } & Retired & 4 & 0 \\
\cline { 2 - 4 } & Student & 0 & 1 \\
\hline \multirow{4}{*}{$\begin{array}{l}\text { Indication } \\
\text { for surgery }\end{array}$} & SLAC arthritis & 11 & 0 \\
\cline { 2 - 4 } & SNAC arthritis & 3 & 0 \\
\cline { 2 - 4 } & $\begin{array}{l}\text { Perilunate } \\
\text { dislocation }\end{array}$ & 0 & 4 \\
\cline { 2 - 4 } & Fracture & 0 & 0 \\
\cline { 2 - 4 } & Mb Kienböck & 1 & 0 \\
\hline \multirow{5}{*}{$\begin{array}{l}\text { Duration of } \\
\text { symptoms }\end{array}$} & $<5$ years & 6 & 0 \\
\cline { 2 - 4 } & $>5$ years & 9 & 0 \\
\hline
\end{tabular}

Abbreviations: SLAC, scaphoLunate advanced collapse; SNAC, scaphoid nonunion advanced collapse.

Table 2 Morphological data from the PIN of patients with wrist arthritis and healthy controls

\begin{tabular}{|l|l|l|}
\hline Variables & Patients $(\boldsymbol{n}=\mathbf{1 5})$ & Controls $(\boldsymbol{n}=5)$ \\
\hline Fascicular & 0.081 & 0.059 \\
area $\left(\mathrm{mm}^{2}\right)$ & $(0.064-0.101)$ & $(0.042-0.13)$ \\
\hline Myelinated fiber & 8,059 & 8,083 \\
density $\left(\mathrm{no} / \mathrm{mm}^{2}\right)$ & $(6,459-10,078)$ & $(2,595-10,619)$ \\
\hline Myelinated fiber & 0.007 & 0.007 \\
diameter $(\mathrm{mm})$ & $(0.006-0.008)$ & $(0.006-0.007)$ \\
\hline
\end{tabular}

Abbreviation: PIN, posterior interosseous nerve.

Note: data are presented as median (interquartile range).

fiber diameter were observed. No signs of inflammation were present in the control group.

\section{Patients}

No general pathological findings in the PIN were observed among the patients with wrist osteoarthritis. Except for three cases in whom no pathological changes were noted, a variety of pathological changes and in various combinations were seen; Clusters of regenerating nerve fibers and abnormally thin myelin sheaths in relation to fiber diameter (six cases), abnormally thin myelin sheaths in relation to fiber diameter without clusters of regenerating nerve fibers (two specimens), perineurial thickening and the presence of mucoid degeneration (six and five specimens, respectively) and endoneural fibrosis (three cases). One specimen exhibited a markedly low fiber density $\left(2,057 / \mathrm{mm}^{2}\right)$. There were no inflammatory changes in PINs from the osteoarthritic wrists. 

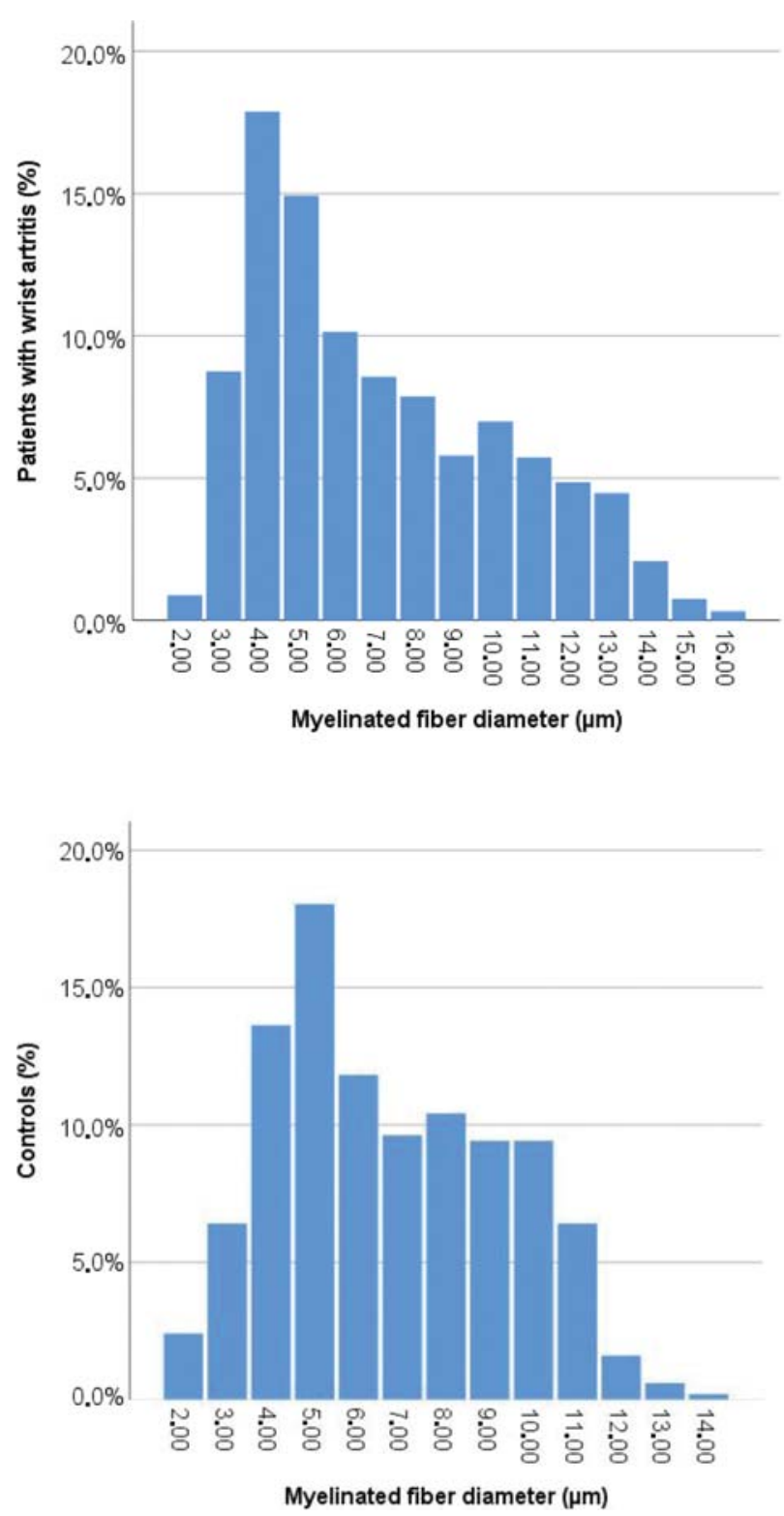

Fig. 2 Size distribution of myelinated fibers in the posterior interosseous nerve of patients with painful wrist arthritis $(n=15)$ and healthy controls $(n=5)$.

\section{Discussion}

\section{Background and General Results}

PIN resection is a method for treating wrist pain caused by for example primary or posttraumatic osteoarthritis. ${ }^{6,7}$ Histopathological changes in PIN at wrist level has been reported in patients with pain after repetitive dorsiflexion maneuvers of the wrist such as during typing and gymnastics $^{11}$ and after wrist sprains, ${ }^{7}$ indicating that structural changes may occur in the PIN after stress. Carr and Davis ${ }^{11}$ speculated that the posttraumatic nerve changes might contribute to wrist pain.

We hypothesized that PINs from osteoarthritic wrists would show morphological changes that would not be present in control wrists without osteoarthritis. However, in the present study no significant differences in parameters commonly used to describe morphology such as myelinated fiber density, fascicular sectional area, or fiber diameter was found between controls and patients with wrist osteoarthritis. We could not observe a specific pattern of histopathology among the patients.

\section{Morphological Appearance of the PIN in Comparison to Previous Studies}

The morphometric appearance of the normal PIN has been described in healthy controls in previous studies. ${ }^{12-16}$ These studies reported a mean fascicular sectional area ranging from 0.084 to $0.12 \mathrm{~mm}^{2,13-16}$ and a myelinated fiber density of 6,128 to $9,256 / \mathrm{mm}^{2}$, which is consistent with the results of both patients and controls in the present study. Osman et al ${ }^{16}$ reported a median myelinated fiber diameter of $7.3 \mu \mathrm{m}$ which is comparable to our results. Osman et $\mathrm{al}^{16}$ and Mojaddidi et $\mathrm{al}^{15}$ quantified the myelinated fiber density and myelinated fiber diameter by electron microscopy at a considerably greater magnification. Our results might not be entirely comparable, since we performed the quantifications at a lower magnification, and therefore might have missed the smallest myelinated fibers. We did not observe any signs of inflammation in the PIN and these have not been described in earlier studies either. ${ }^{12-16}$

\section{Pathological Changes among Patients and Controls}

PINs from both groups contained a few samples where a markedly low myelinated fiber density was noticed compared with previous studies. ${ }^{13-16}$ Further, round amorphous intraneural bodies of unknown origin were found in two controls and two patients (-Fig. 1B, D). Their appearance was not typical for the Renaut bodies ${ }^{17}$ but they stained positive for epithelial membrane antigen (EMA) indicating a perineural origin and the structure had similarities with intraneural perineuroma.

Pathological changes such as perineurial thickening, loss of myelinated fibers, presence of mucoid degeneration and abnormally thin myelin sheaths in relation to fiber diameter were seen in several specimens from both groups. In accordance to our findings, Carr and Davis ${ }^{11}$ found perineurial thickening and fibrosis, axonal degeneration, and the presence of fibrinoid intraneural bodies in patients suffering from wrist pain after repetitive dorsiflexion of the wrist. Dellon $^{7}$ reported epineural and perineural fibrosis in patients with pain 6 months or more after wrist sprains with normal radiographs. Similar morphological changes such as demyelination with loss of fibers and endoneural and perineural fibrosis has also been described in workers exposed to vibration. ${ }^{12}$

\section{Pathological Changes among Control Subjects}

Surprisingly, varying degrees of histopathological changes were found in the controls who were healthy, asymptomatic, and significantly younger than the patients with osteoarthritic wrist changes. Hence, we expected this group to present normal nerves, with no histopathological changes due to age, stress, or osteoarthritis. None of the control subjects had experienced any previous wrist trauma that 
could explain the morphological changes in the PIN, but three out of five participated regularly in sports or other activities where the wrist was exposed to a high workload (competitive arm wrestling, motor cross, or show jumping). Perhaps the structural changes in the PIN occur ahead of symptoms and may be associated not only with repetitive workloads and minor sprains, as reported in previous studies, ${ }^{7,11}$ but also with demanding wrist activity such as heavy workloads or demanding sports.

Our findings of histopathological changes among both patients and controls are consistent with the findings of Bourke et $\mathrm{al}^{18}$ who observed nerve enlargement, vessel hyalinization, and perineural and endoneural fibrosis in asymptomatic autopsy controls that were compared with patients suffering from Morton's neuroma. In a study by Osman et $\mathrm{al}^{16}{ }^{16}$ three out of nine control PINs from patients with carpal tunnel syndrome, exhibited some pathology such as a few myelin debris, demyelination of a few fibers, and the presence of remyelinated axons. These findings suggest that structural nerve changes of unknown significance can exist in asymptomatic subjects.

There appears to be no apparent difference between patients suffering from wrist osteoarthritis and healthy controls regarding morphometrical data. Therefore, we believe that the structural nerve changes in the PIN are unlikely to contribute to the symptoms of pain. More likely, the pain originates in the wrist and is only conducted by the nerve.

\section{Limitations}

There are several limitations to this study. Since the number of nerve fibers are known to decrease progressively with age $^{19}$ and the subjects in our control group are significantly younger than the patients with wrist osteoarthritis, the groups might not be entirely comparable. Due to the younger age, we expected the control group to have a more normal morphology than the patients. However, we did not find any differences in fiber density or mean fascicular sectional area between the groups. Furthermore, the controls might not be representative of the general population, since three out of five are manual workers and they are all highly active individuals whose wrists might be exposed to a higher workload than the general population.

It can be questioned whether the nerve changes observed in the control group were caused by the trauma. However, it seems unlikely that the observed changes would have developed in less than 1 week.

Jariwala et $\mathrm{al}^{20}$ and Carr and Davis ${ }^{11}$ demonstrated that the diameter of PIN increases distally. In our study, some samples might have been collected at slightly different levels, which might have influenced the fascicular area and fiber density measurements. Further, measurements may be somewhat distorted since quantification of myelinated axons was performed in areas where all fibers had a circular or ovoid form and not in the whole fascicle. Hence, the measured area might not be entirely representative of a nerve fascicle with a lot of fibrosis resulting in a false high fiber density. Furthermore, the specimens are not analyzed by electron microscopy to detect changes in unmyelinated fibers.

\section{Conclusion}

Since we investigated a small number of control subjects, we might have selected the pathological ones by chance. We cannot draw any conclusions about normal PIN morphology from our small sample of nonsymptomatic wrists. Further studies of the normal histological appearance of the terminal PIN nerve are needed. Also, additional studies of pathological changes in the distal PIN nerve among manual workers would be of interest.

Note

The work was performed at the department for Hand Surgery (Handkirurgiska kliniken) Södersjukhuset.

Ethical Approval

The study was approved by the Regional Ethic committee.

Funding

This work was supported by AFA (Arbetsmarknades Försäkrings Aktiebolag) research fund. Funding for clinical research was provided at Karolinska Institutet at Södersjukhuset and Värkstadsstiftelsen.

Conflict of Interest

None declared.

\section{References}

1 Wilhelm A. [Innervation of the joints of the upper extremity]. Z Anat Entwicklungsgesch 1958;120(05):331-371

2 Wilhelm A. [Articular denervation and its anatomical foundation. A new therapeutic principle in hand surgery. On the treatment of the later stages of lunatomalacia and navicular pseudarthrosis]. Hefte Unfallheilkd 1966;86:1-109

3 Fukumoto K, Kojima T, Kinoshita Y, Koda M. An anatomic study of the innervation of the wrist joint and Wilhelm's technique for denervation. J Hand Surg Am 1993;18(03):484-489

4 Ferreres A, Suso S, Ordi J, Llusa M, Ruano D. Wrist denervation. Anatomical considerations. J Hand Surg [Br] 1995;20(06):761-768

5 Van de Pol GJ, Koudstaal MJ, Schuurman AH, Bleys RL. Innervation of the wrist joint and surgical perspectives of denervation. J Hand Surg Am 2006;31(01):28-34

6 Ferreres A, Suso S, Foucher G, Ordi J, Llusa M, Ruano D. Wrist denervation. Surgical considerations. J Hand Surg [Br] 1995;20 (06):769-772

7 Dellon AL. Partial dorsal wrist denervation: resection of the distal posterior interosseous nerve. J Hand Surg Am 1985;10(04): 527-533

8 Buck-Gramcko D. Wrist denervation procedures in the treatment of Kienböck's disease. Hand Clin 1993;9(03):517-520

9 Xiong L, Harhaus L, Heffinger C, et al. A comparative study on autologous bone grafting combined with or without posterior interosseous nerve neurectomy for scaphoid nonunion treatment. J Plast Reconstr Aesthet Surg 2015;68(08):1138-1144

10 Loh YC, Stanley JK, Jari S, Trail IA. Neuroma of the distal posterior interosseous nerve. A cause of iatrogenic wrist pain. J Bone Joint Surg Br 1998;80(04):629-630

11 Carr D, Davis P. Distal posterior interosseous nerve syndrome. J Hand Surg Am 1985;10(6 Pt 1):873-878 
12 Strömberg T, Dahlin LB, Brun A, Lundborg G. Structural nerve changes at wrist level in workers exposed to vibration. Occup Environ Med 1997;54(05):307-311

13 Thomsen NO, Mojaddidi M, Malik RA, Dahlin LB. Biopsy of the posterior interosseous nerve: a low morbidity method for assessment of peripheral nerve disorders. Diabet Med 2009;26(01):100-104

14 Thomsen NO, Mojaddidi M, Malik RA, Dahlin LB. Reduced myelinated nerve fibre and endoneurial capillary densities in the forearm of diabetic and non-diabetic patients with carpal tunnel syndrome. Acta Neuropathol 2009;118(06):785-791

15 Mojaddidi MA, Ahmed MS, Ali R, et al. Molecular and pathological studies in the posterior interosseous nerve of diabetic and nondiabetic patients with carpal tunnel syndrome. Diabetologia 2014;57(08):1711-1719

16 Osman AA, Dahlin LB, Thomsen NO, Mohseni S. Autophagy in the posterior interosseous nerve of patients with type 1 and type 2 diabetes mellitus: an ultrastructural study. Diabetologia 2015;58 (03):625-632

17 Jefferson D, Neary D, Eames RA. Renaut body distribution at sites of human peripheral nerve entrapment. J Neurol Sci 1981;49(01): 19-29

18 Bourke G, Owen J, Machet D. Histological comparison of the third interdigital nerve in patients with Morton's metatarsalgia and control patients. Aust N Z J Surg 1994;64(06):421-424

19 Bilbao JM, Anthony DC, De Girolami U. Methods for examination of peripheral nerve. In: Vallant J-M, Weiss J, eds. Peripheral Nerve Disorders: Pathology and Genetics. 1st ed. John Wiley \& Sons, Incorporated; 2014:24-29

20 Jariwala A, Krishnan B, Soames R, Wigderowitz CA. Important anatomical relationships of the posterior interosseous nerve in the distal forearm for surgical planning: a cadaveric study. J Wrist Surg 2014;3(01):60-63 